\title{
Temperature-modulated thermomechanical analysis as a potential technique for irreversible stress relaxation measurement in various cables
}

\author{
Mateusz Lutomski $^{1,2}$ (D) Barbara Gawdzik ${ }^{1} \cdot$ Beata Podkościelna $^{1}$
}

Received: 9 November 2015/ Accepted: 7 June 2016/Published online: 21 June 2016

(c) The Author(s) 2016. This article is published with open access at Springerlink.com

\begin{abstract}
Temperature-modulated thermomechanical analysis (TMA) is a technique which allows for separation of the temperature-dependent thermal expansion from the time and temperature-dependent creep or stress relaxation behavior. Extrusion during the cable production process may orient and stretch polymer chains which are rapidly cooled in water. Such frozen-in stresses might relax over a longer time period, or when the sample is heated up, the polymer chains are able to move back to their initial state. Using this method allows for measurement of both effects-irreversible stress relaxation and reversible thermal expansion-at the same time. Additionally, a reversible signal might be used for the determination of the coefficient of thermal expansion for different materials. This work compares polyethylene samples with and without frozen-in stresses measured on standard and temperature-modulated TMA.
\end{abstract}

Keywords mT-TMA · Shrink-back · Shrinkage · Thermomechanical analysis $\cdot$ Optical cables

Mateusz Lutomski

Mateusz.lutomski@corning.com

1 Department of Polymer Chemistry, Faculty of Chemistry, Maria Curie-Sklodowska University, pl. Maria CurieSklodowskiej 3, 20-031 Lublin, Poland

2 Corning Optical Communications, Smolice 1E, Stryków, Poland

\section{Introduction}

Optical cables and fibers are extremely sensitive to mechanical, thermal and environmental conditions, which can affect their optical performance. Shrinkage and dimensional length changes which are a consequence of thermal cycling can reduce those properties significantly. Even though most materials expand with increasing temperature and decrease during cooling, more irreversible mechanisms might be observed which occur in the opposite direction. For example, long molecule chains of polymer stretched out by the extrusion process and frozen in that state by rapid cooling may relax during heating the sample so that the sample will expand and shrink back at the same time. The expansion could be separated into the reversing length change, while the stretching is resolved into a non-reversing dimension change. The thermodynamic and kinetic components in this case are in the opposite direction, and the total length change might be not much different from the initial length. A further cooling cycle will add another shrinking effect—physical contraction, which will further change the sample diameters; higher shrinkage will occur after second thermal cycle [1]. Thermomechanical analysis with a modulated temperature program (mT-TMA) seems to be a potential technique for the measurement of all components of shrinkage including the total length change in the sample and the deconvoluted reversible and irreversible parts of dimensional change during temperature cycling. Such novel approach may simplify the analysis of shrink-back experiments results and may reduce measurement cycles to only one heating.

\section{The technique}

In the first instance, a modulated temperature program was applied to differential scanning calorimetry (DSC) and also 
other techniques including thermogravimetry (TG) [2], dynamic mechanical analysis (DMA) [4] and finally TMA $[5,6]$. The common idea for those techniques is changing the linear heating rate into sinusoidal (Fig. 1).

Depending on the equipment type for DSC, temperature modulation might be controlled by the block temperature, reference or sample thermocouples. In the next step, discrete Fourier transformation is applied in real time to continuously determine the average value and the amplitude value for each signal. While modulated DSC involves deconvolution of the heat flow rate into the part which follows modulation (reversing) and one independent-irreversible [3], in mT-TMA, the sample length $(L)$ change is measured. The rate of change in the sample length with respect to time $(\mathrm{d} L / \mathrm{d} t)$ can be divided into two components Eq. 1:

$\mathrm{d} L / \mathrm{d} t=\mathrm{d} L / \mathrm{d} T \cdot \mathrm{d} T / \mathrm{d} t+f^{\prime}(t, T)$

where $L$, length; $t$, time; $T$, temperature; $\mathrm{d} L / \mathrm{d} T$, the thermal expansion coefficient; $\mathrm{d} T / \mathrm{d} t$, heating rate; $f^{\prime}(t, T)$, encompass changes in length which occur due to relaxation of stresses in the sample, or deformation under the applied load [5]. The amplitudes of length and temperature (reversible part), as well as the average length (total length) (Fig. 2) are obtained from the Fourier transformation process. $\int \mathrm{d} T / \mathrm{d} t K K$ is the underlying heating rate averaged over a single period, while $K$ is a calibration constant close to unity. Finally, the irreversible part, which describes stress relaxation (or sample deformation), is obtained from the difference between the total and reversing contributions $[3,4]$. Authors also describe measurement settings which affect the final results and special calibration techniques [3, 5-7]. Calibration of modulated TMA includes tests on Al slug, cell constant calibration and comparison of CTE results of standard and modulated measurements.

\section{Shrink-back phenomena and coefficient of thermal expansion}

Shrink-back is a type of polymer dimensional change, which occurs because polymer chains that tend to be tangled have been stretched out by the extrusion process and frozen in that state by rapidly cooling the stretched polymer [8]. The problem starts when the temperature is increased and polymer chains become mobile enough to revert to their more entangled configuration. This results in a permanent shrinkage of the material which can be additionally increased by thermal contraction during the cooling cycle.

Thermal expansion is the tendency of matter to change in volume in response to a change in temperature. The degree of expansion divided by the change in temperature is called the material's coefficient of thermal expansion and generally varies with temperature. When heat is added to most materials, the average amplitude of the atoms vibrating within the material increases. This effect increases the separation between the atoms, causing the material to expand. If the material does not go through a phase change, the expansion can be easily related to the temperature change [9]. If the linear coefficient of thermal expansion is known, the change in the sample length can be theoretically calculated for each degree of temperature change. From the theory, this effect should also work in
Fig. 1 TMA linear and modulated temperature program

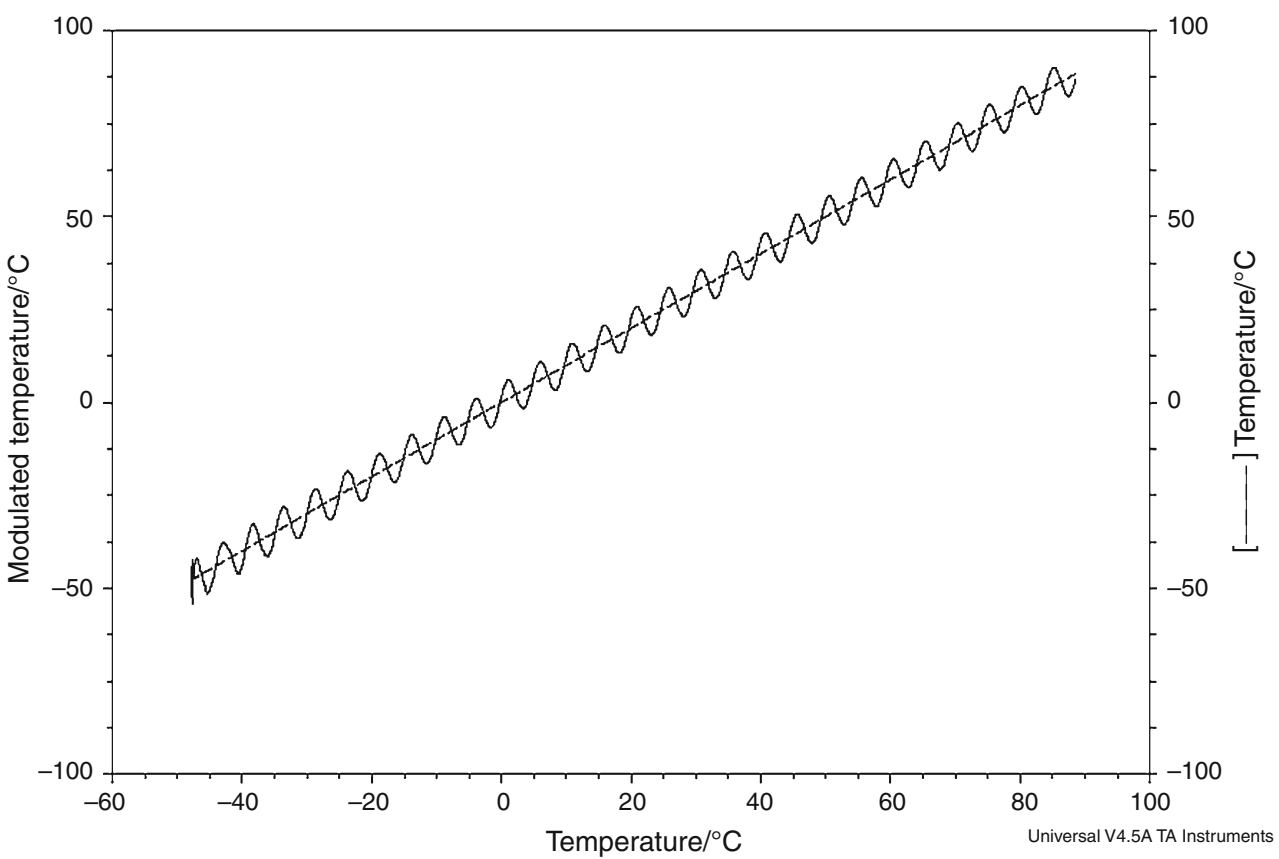




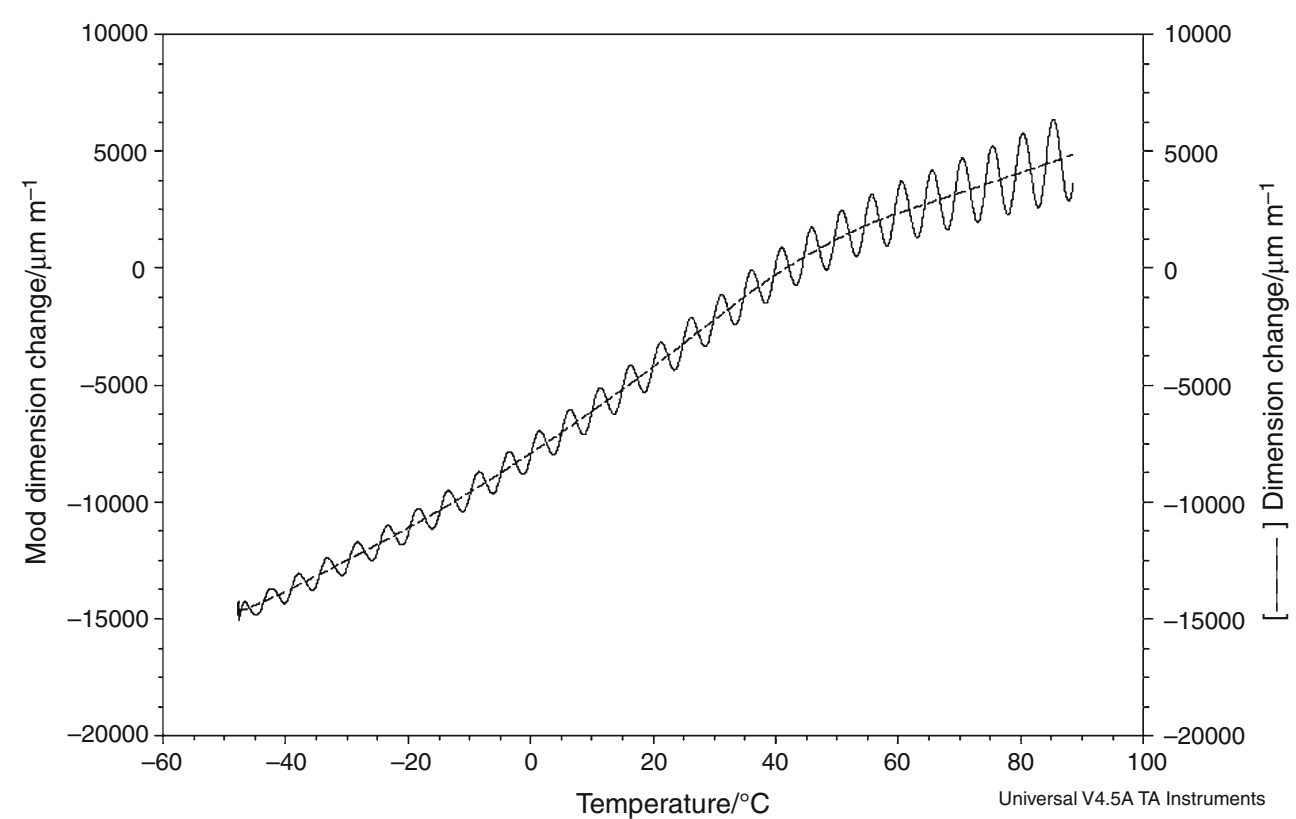

Fig. 2 Modulated dimension change versus dimension change (polyethylene sample)

reverse. If energy is removed from a material, then the sample temperature will decrease, causing the contraction of the entire sample. The coefficient of thermal expansion can serve as a material characteristic only where material behavior is reversible. Unfortunately, it does not work for polymers with frozen-in stresses and irreversible effects related with chain reorganization. The metastable nature of most oriented polymers causes the relaxation to the disordered state and will not be reversible until structural equilibrium is achieved.

\section{Experimental}

For two methods comparison, standard TMA and modulated TMA measurements were performed on polyethylene (PE) samples. For clear extrusion influence on shrink-back phenomena, two different sets of samples were prepared. One sample was collected from extruded cable jacket ("PE Cable"), which should release the frozen-in stresses during the first heating cycle. The second sample was prepared directly from raw material using temperature press and standard settings (IEC 60811-4-1) for PE samples preparation ("PE Plate").

Differences in irreversible dimension change during the first heating cycle were the focus of the experiment. Also, the Coefficient of thermal expansion was compared for both samples with standard and modulated measurement.

The measurements were performed on TA Instruments mT-TMA model Q400. The program started at $-50{ }^{\circ} \mathrm{C}$ with heating/cooling rate $2{ }^{\circ} \mathrm{C} \min ^{-1}$ up to $100{ }^{\circ} \mathrm{C}$, cooling back to $-50{ }^{\circ} \mathrm{C}$ and heating up to $90{ }^{\circ} \mathrm{C}$. For easier results comparison, starting points at $-50{ }^{\circ} \mathrm{C}$ are $0 \%$ of dimension change (Fig. 3).

\section{Results and discussion}

The PE sample taken from a plate (solid line) reveals similar dimensional changes during heating and cooling cycles (around $3 \%$ ). There is no extrusion influence, and the total signal is mostly caused by reversible linear extension and contraction. The signal from the extruded sample ("PE Cable") has different shape and dimensional change range. Instead of expanding in a similar way to Sample "PE Plate", shrink-back starts to occur around $40{ }^{\circ} \mathrm{C}$ during the heating cycle, where the relaxation of polymer chains begins. Expected expansion is reduced and would cause much higher shrink after an additional cooling cycle. Measurements have been performed with extension probes, calibrated with aluminum reference sample with MCA 70 cooling accessory.

Similar measurements were performed on the same PE samples, using temperature modulation and total length signal deconvolution to the reversible and irreversible parts. Figure 4 confirms that the total length change signal for "PE plate" is almost identical with the reversible part obtained from modulated measurement. The low value of the irreversible part $(0.47$ and $-0.20 \%)$ is probably caused by the influence of raw material production or plate preparation. Figure 5 shows the extruded sample "PE Cable", where the irreversible signal is much more significant and stress relaxation occurs around $40{ }^{\circ} \mathrm{C}$ in much 
Fig. 3 Standard TMA measurement (total dimension change of two PE samples"PE Cable" and "PE Plate")
Fig. 4 Temperature modulated TMA measurement of sample "PE plate"
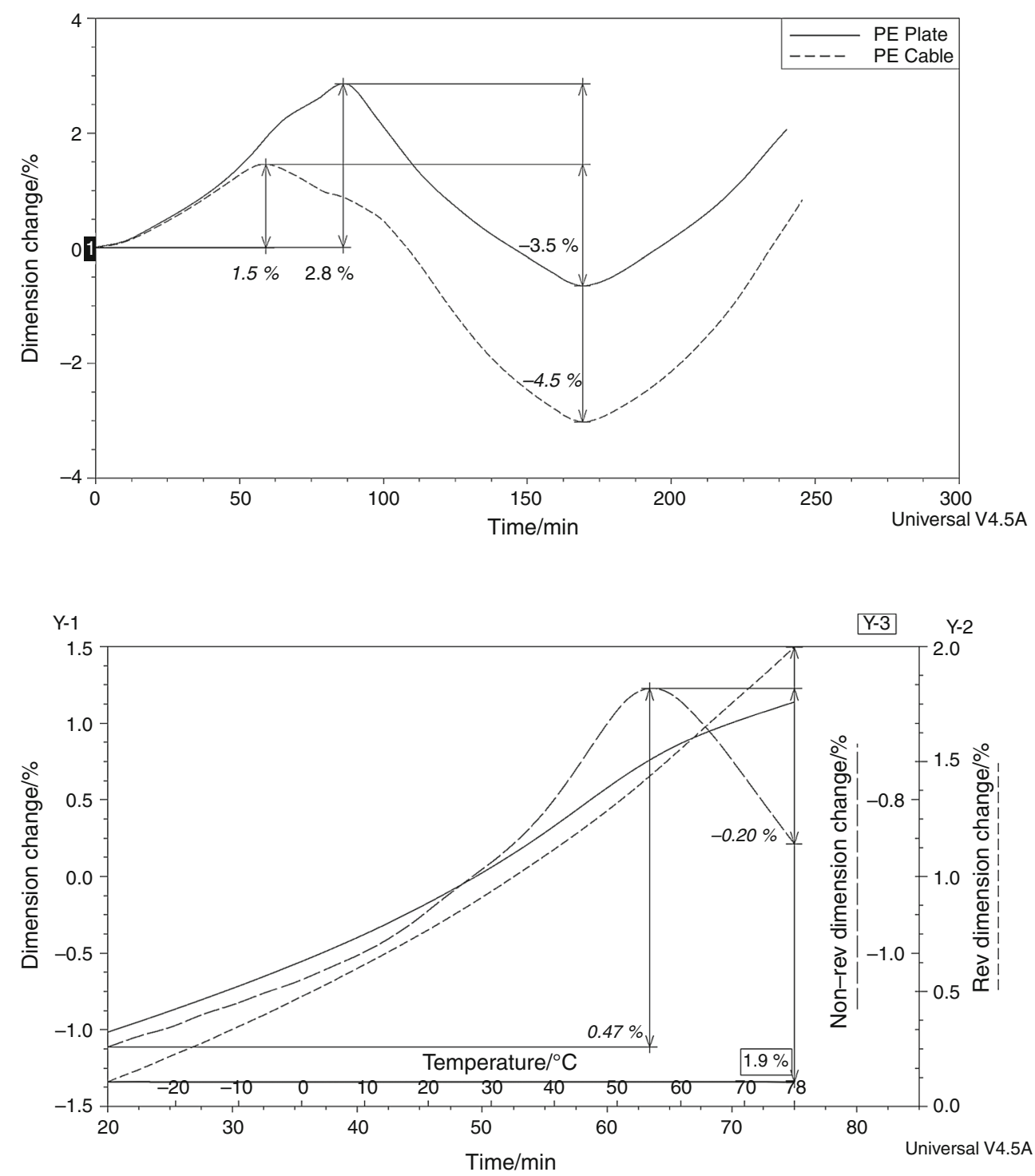

greater scale. Measured shrink-back in this case is a couple of times higher, comparing to the previous sample $(1.3 \mathrm{vs}$ $0.2 \%)$. When compared to the difference between the expansion of two samples from Fig. 3, the shrink-back value from the irreversible signal seems to have an almost identical value $(2.8-1.5 \% \approx 1.3 \%)$ and shows how much stress relaxation decreases sample expansion. Reversible change is at a similar level for both samples (1.8 and $1.9 \%$ ) (Fig. 6).

\section{CTE}

From the reversible signals, it is also possible to calculate the coefficient of linear expansion-Alpha, a derivative of the reversible signal with respect to temperature. International standards also describe how to calculate it from the standard measurement (e.g., Coefficient of Linear Thermal Expansion ASTM E831, ASTM D696, ISO 11359). To reduce the influence of irreversible effects, results from the second heating cycles could be taken at a specific temperature, with the assumption that all stresses and irreversible signals would be erased during the first heating and cooling. The comparison of the two methods is also presented in this work, using the previously described samples. All results are compared in Table 1.

Data received from the second heating cycle with the standard TMA method seem to be "affected" by the irreversible part, as the value for the cable sample is much higher compared to the "PE Plate" sample. Results from the mT-TMA are similar for both samples (6\% difference), while the CTE values taken from the second cycle of the standard TMA technique vary around $28 \%$. Modulated measurement for the sample with oriented polymer chains seems to be more reliable as the values of CTE are at the same range and fit the theoretical CTE for polyethylene ( 100-200 $\times 10^{-6} \mathrm{~m} \mathrm{~m}^{-1} \mathrm{~K}^{-1}$ ) although changing, for 
Fig. 5 Temperature modulated TMA measurement of sample "PE Cable"
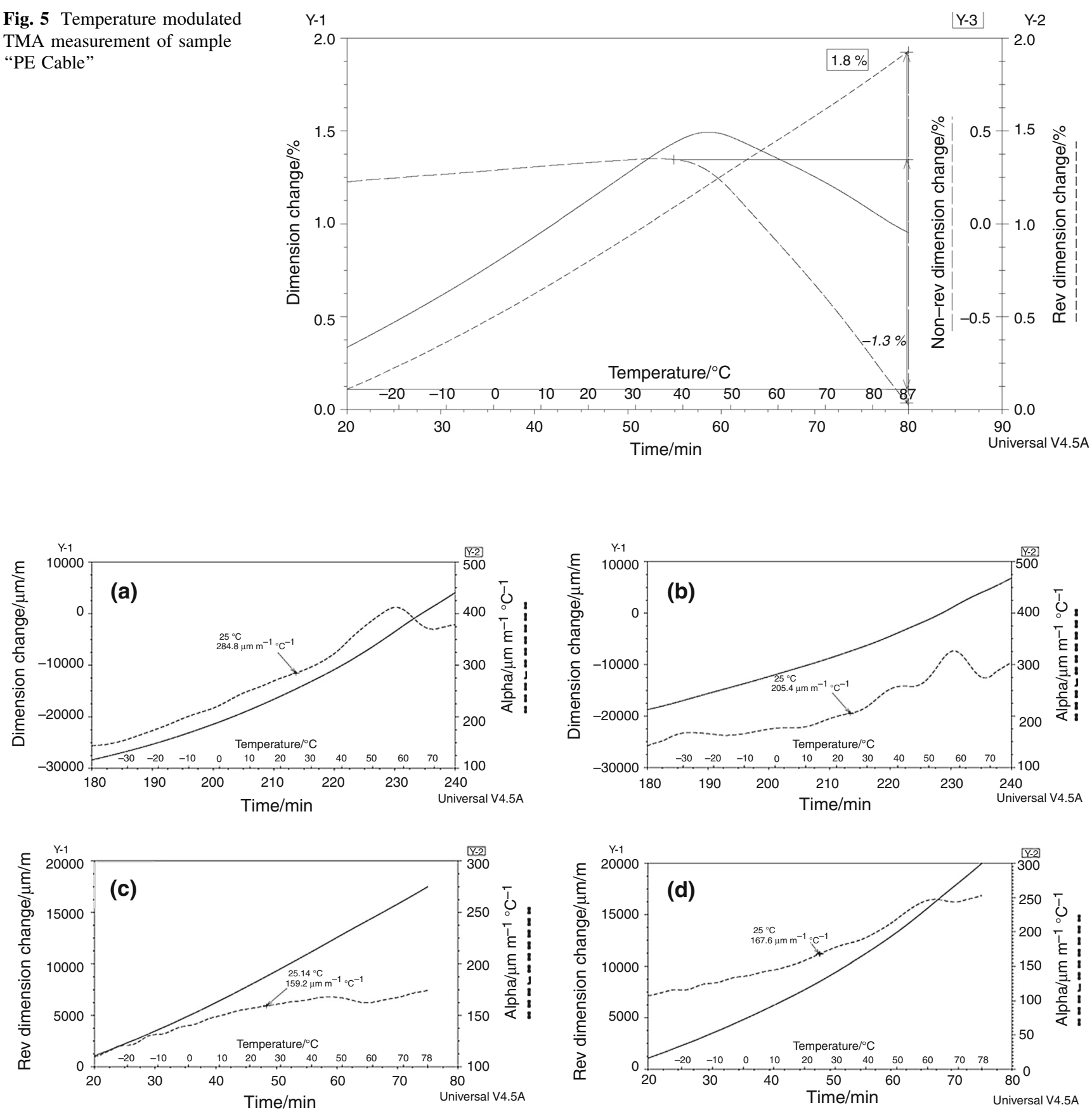

Fig. 6 a CTE of Sample "PE Cable", standard TMA, data from second heating cycle at $25^{\circ} \mathrm{C}$, b CTE of Sample "PE Plate", standard TMA, data from second heating cycle at $25{ }^{\circ} \mathrm{C}$, c CTE of Sample "PE Cable", mT-TMA, data from first heating cycle at $25{ }^{\circ} \mathrm{C}, \mathbf{d}$ CTE of Sample "PE Plate", mT-TMA, data from first heating cycle at $25^{\circ} \mathrm{C}$

Table 1 Comparison of dimension changes and CTE's for samples "PE Plate" and "PE Cable"

\begin{tabular}{|c|c|c|c|c|c|c|}
\hline \multirow[t]{2}{*}{ Sample } & \multicolumn{2}{|c|}{$\mathrm{CTE} / 10^{-6} \mathrm{~m} \mathrm{~m}^{-1} \mathrm{~K}^{-1}$} & \multicolumn{2}{|l|}{ TMA/\% } & \multicolumn{2}{|l|}{ mT-TMA/\% } \\
\hline & TMA & mT-TMA & First expansion & Maximum dimension change & Irreversible signal & Reversible signal \\
\hline "PE plate" & 205.4 & 167.6 & 2.8 & 3.5 & -0.2 & 1.9 \\
\hline "PE cable" & 284.8 & 159.2 & 1.8 & 4.5 & -1.3 & 1.8 \\
\hline
\end{tabular}


example, the heating rate might increase those values. Process parameters also do not affect the CTE value obtained from the mT-TMA reversible signal. Moreover, only one heating cycle was used to get all the results, while standard TMA measurement requires additional cooling and heating cycles to clear the irreversible part. In this approach, taking the data from the second heating cycle runs the risk that not all stress is relaxed during the first heating, due to limited measurement time, heating rate or temperature range (material melting). Also, the first cooling (cooling rate) affects sample crystallinity and may create a new, (much smaller than the original) irreversible signal.

\section{Conclusions}

Based on PE samples and other measurements not described in this work for materials like PBT, PC and flame retardant polymers, it seems that modulated measurements on a thermomechanical analyzer might be a very powerful tool for shrink-back, stress relaxation and other irreversible mechanisms related to postproduction influence. One of the most important advantages of this technique is that all previously described information can be received from only one heating cycle. Shrink-back and CTE results seems to be reliable, especially when compared to the standard, two-cycle TMA. CTE is not affected by process parameters/irreversible signal, and results are similar without sample preparation method influence. In this case, shrinkback analysis from the first mT-TMA heating cycle gives similar information as standard TMA measurement from two samples-the difference between expansions of samples with and without the extrusion process. Completely different information is obtained when the second TMA heating cycle is subtracted from the first one. This may be caused by the fact that the first cycle may not completely clear the thermal history of the sample and/or additional cooling could build a new (much smaller) irreversible signal by changing sample crystallinity. Although modulated measurement usually takes much more time because of lower heating rates(1-5 $\left.\mathrm{K} \mathrm{min}^{-1}\right)$, the total experiment time may still be similar. Taking into consideration that it is possible to perform only one heating measurement in a very specific, narrow range, it may take similar or even less time than three full cycles, with heating rate $10 \mathrm{~K} \mathrm{~min}^{-1}$. However, this assumption depends on the specific material character and measurement purpose. Other factors determining measurement time and results quality such as the influence of measurement settings (modulation amplitude, frequency and heating rate) are still under investigation.

Open Access This article is distributed under the terms of the Creative Commons Attribution 4.0 International License (http://crea tivecommons.org/licenses/by/4.0/), which permits unrestricted use, distribution, and reproduction in any medium, provided you give appropriate credit to the original author(s) and the source, provide a link to the Creative Commons license, and indicate if changes were made.

\section{References}

1. Lutomski M, Gawdzik B. Polymer dimensional changes in optical cables. Ann UMCS Chem. 2014;68(1-2):33-49.

2. Reading M. Modulated differential scanning calorimetry-a new way forward in materials characterisation. Trends Polym Sci. 1993;1:248-53.

3. Wunderlich Bernhard. Thermal analysis of polymeric materials. Berlin: Springer; 2005.

4. Wurm A, Merzlyakov M, Schick C. Temperature modulated dynamic mechanical analysis. Thermochim Acta. 1999;330:121-30.

5. Blaine RL, TA Instruments. Modulated thermomechanical analysis-measuring expansion and contraction simultaneously. http:// www.tainstruments.com/pdf/literature/TA311a.pdf (2003). Accessed 09 Jan 2014.

6. Price DM. Theory and applications of modulated temperature programming to thermomechanical techniques. In: Proceedings of the 28 conference of the North American Thermal Analysis Society, October 4-6, 2000, Orlando, Florida. http://www.sump4. com/publications/conf016.pdf.

7. Price DM. Novel methods of modulated-temperature thermal analysis. Thermochim Acta. 1998;315:11-8.

8. Hurley W, Freeland MR, Ellwanger MR. Shrinkback attenuation in loose tube cables. Corning Cable Systems, Hickory, NC, IWCS paper. Nov. 2007, Lake Buena Vista, FL, USA, pp. 414-418.

9. Callister WD, Rethwisch DG. Materials science and engineering: an introduction. 9th ed. Wiley: New York; 2014. 